\title{
Levantamento das espécies exóticas arbóreas e seu impacto nas espécies nativas em áreas adjacentes a depósitos de resíduos domiciliares
}

\author{
Otacílio Antunes Santana* \\ José Imaña Encinas \\ Universidade de Brasília, Departamento de Engenharia Florestal \\ *Autor para correspondência \\ Rua 16-A, 841, Setor Aeroporto CEP: 74075-150, Goiânia - GO, Brasil \\ otaciliosantana@gmail.com
}

Submetido em 07/02/2008

Aceito para publicação em 02/07/2008

\section{Resumo}

Os objetivos deste trabalho, em áreas adjacentes à deposição de resíduos domiciliares, foram: i) realizar o levantamento das espécies exóticas, ii) verificar o seu impacto nas espécies nativas arbóreas de Cerrado, e iii) comparar os resultados com áreas controles. Vinte e duas espécies exóticas foram encontradas nas parcelas estudadas próximos aos depósitos de resíduos domiciliares, distribuídas entre treze famílias. Apenas duas espécies foram encontradas nas áreas controle. A presença de depósitos de resíduos domiciliares aumenta a incidência de espécies exóticas arbóreas, causando um gradiente na distribuição de seus indivíduos, padrão não observado nas áreas controles.

Unitermos: invasão biológica, impacto ambiental, espécies exóticas

\section{Abstract}

Survey of exotic tree species and their impact on native species in areas adjacent to landfills. This work aimed to i) carry out a survey of exotic tree species, ii) observe their impact on Cerrado native species, and iii) compare the results with control areas. Twenty-two exotic species were sampled in the studied plots near landfills that were shared by thirteen families. Just two species were sampled in the control areas. Landfills enhance the incidence of exotic species. This causes a gradient of distribution in the individuals, a standard which is not observed in the control areas.

Key words: biological invasion, environmental impact, exotic species

\section{Introdução}

Contaminação biológica é o processo de introdução e adaptação de espécies que não fazem parte naturalmente de um dado ecossistema, mas que se naturalizam e passam a provocar mudanças em seu funcionamento
(Ziller, 2000). A introdução pode ser realizada intencional ou acidentalmente, por vias humanas ou não (Gotelli e Colwell, 2001).

Ao contrário de muitos problemas ambientais que se amenizam com o tempo, como por exemplo, a 
poluição química, a contaminação biológica tende a se multiplicar constantemente, causando problemas de longo prazo que se agravam com o passar do tempo, e não permitem que os ecossistemas afetados se recuperem naturalmente (Westbrooks, 1998).

Trata-se de espécies que se estabelecem em novos territórios nos quais proliferam, dispersam e persistem em detrimento de espécies próprias de ecossistemas nativos, causando alterações nos processos ecológicos (Mack et al., 2000).

Algumas características que permitem que as espécies exóticas se tornem potenciais invasoras são: alta taxa de crescimento relativo, grande produção de sementes pequenas e de fácil dispersão, alta longevidade das sementes no solo, alta taxa de germinação dessas sementes, maturação precoce das plantas já estabelecidas, floração e frutificação mais prolongadas, alto potencial reprodutivo por brotação, pioneirismo, alelopatia e ausência de inimigos naturais (Genovesi, 2005; Parker et al., 1999).

Inúmeros termos têm sido empregados para definir espécies exóticas: não nativas, invasoras, alienígenas, daninhas, introduzidas, não-aborígines, não-indígenas, nocivas, naturalizadas, pragas, pragas ambientais, pragas florais, pragas de áreas naturais e alóctones (Westbrooks, 1998; Randall, 1996).

Gotelli e Colwell (2001) observaram que em áreas ambientais protegidas de ações antrópicas e em estações ecológicas, alguns indivíduos de espécies de plantas exóticas surgiram em meio à vegetação nativa, devido principalmente à sua forma de dispersão. Eles constataram também, o que foi corroborado por Lima (2003), que em áreas perturbadas antropicamente, ou em vegetação de bordas de cidades há uma crescente disseminação das espécies exóticas.

Pereira e Filgueiras (1988), com trabalho desenvolvido em área de Cerrado strictu sensu, sobre problemas decorrentes da invasão biológica, encontraram um total de 252 espécies exóticas dentro da Reserva Ecológica do IBGE, em Brasília.

Das 44 gramíneas africanas introduzidas no Brasil, onze apresentam elevado grau de agressividade e invasão (Filgueiras, 1990 e 2005).
$\mathrm{Na}$ África do Sul, também em regiões de savana, estima-se um total de 491 espécies exóticas (IUCN, 2000). A Nova Zelândia conta atualmente com cerca de 20 mil espécies introduzidas, mais de $70 \%$ para fins ornamentais (IUCN, 2000). Nos Estados Unidos, estimase a introdução de mais de 4.600 espécies exóticas às ilhas havaianas, 1.045 na Califórnia e 1.180 na Flórida (Westbrooks, 1998).

Algumas espécies arbóreas exóticas já foram relatadas com alto grau de disseminação, como é o caso das espécies de Pinus e Eucalyptus no Brasil, se destacando nas invasões por altos valores da área basal em relação à vegetação nativa (Zancheta e Diniz, 2006).

Os objetivos deste trabalho, em áreas adjacentes à deposição de resíduos domiciliares, foram: i) realizar o levantamento das espécies exóticas, ii) verificar o seu impacto nas espécies nativas arbóreas de Cerrado, e iii) comparar os resultados com áreas controles.

\section{Materiais e Métodos}

Duas áreas próximas a depósitos de resíduos domiciliares foram escolhidas por estarem cobertas com vegetação nativa: uma no Parque Nacional de Brasília, distante a $15 \mathrm{~m}$ do aterro do Jockey Club de Brasília $\left(15^{\circ} 45^{\prime} 56^{\prime}\right.$ 'S e $\left.47^{\circ} 59^{\prime} 55^{\prime \prime} \mathrm{W}\right)$, denominada BSB; e outra também a $15 \mathrm{~m}$ do aterro Sanitário de Goiânia (16³9'09"S e 49²3’37’W), denominada GYN, localizada na Chácara São Joaquim/GO. Para a amostragem controle, outras duas áreas foram estabelecidas: uma no próprio Parque Nacional de Brasília, distante $15 \mathrm{~km}$ do aterro (BSB - Controle: $15^{\circ} 38^{\prime} 11^{\prime \prime} \mathrm{S}$ e $\left.48^{\circ} 01^{\prime} 55^{\prime} \mathrm{W}\right)$; e outra em Goiânia na área do Campus II da Universidade Católica de Goiás (GYN - Controle: $16^{\circ} 37^{\prime} 48^{\prime \prime}$ 'S e 49 $12^{\circ} 46^{\prime \prime} \mathrm{W}$ ), distante também, aproximadamente, a $15 \mathrm{~km}$ do aterro.

$\mathrm{O}$ aterro do Jockey Club de Brasília foi criado e implementado há 35 anos, e atualmente recebe cerca de 1.800 toneladas diariamente de resíduos domiciliares (Seduh, 2007). O aterro Sanitário de Goiânia entrou em funcionamento em 1983 e atualmente recebe, por dia, cerca de 1.200 toneladas de resíduos domiciliares (Prefeitura de Goiânia, 2007). 
As áreas escolhidas situam-se na região CentroOeste com o clima Aw em Brasília e Cwa em Goiânia de acordo com a classificação de Köppen. A pluviosidade anual e temperatura média anual são de $1.440 \mathrm{~mm}$ e $22^{\circ} \mathrm{C}$, respectivamente, para as duas áreas. Duas estações são definidas para estes climas: úmida e chuvosa, de outubro a março, e seca, de abril a setembro.

As altitudes das áreas de amostragem foram: BSB 797m; BSB - Controle 838m; GYN 1.115m e GYN Controle $1.150 \mathrm{~m}$. As declividades das parcelas nas áreas amostradas não ultrapassam a 15\%, correspondentes a um relevo suave ondulado.

Os solos nas regiões estudadas, tanto em Goiânia como em Brasília, foram classificados de Latossolos vermelho-escuros, segundo o Sistema Brasileiro de Classificação dos Solos (Embrapa, 1999; Santos et al., 2003).

A classe textural da análise granulométrica das duas áreas estabelecidas foi classificada como argilosa, com valores aproximados de $380 \mathrm{~g} \cdot \mathrm{kg}^{-1}$ para argila, $182 \mathrm{~g} \cdot \mathrm{kg}^{-1}$ para silte e $438 \mathrm{~g} \cdot \mathrm{kg}^{-1}$ para areia, com diâmetro médio ponderado de $2,81 \mathrm{~mm}$, apresentando alta taxa de infiltração hídrica, cerca de $90 \mathrm{~cm} . \mathrm{h}^{-1}$ (Santana e ImañaEncinas, 2004).

As quatro áreas amostradas estão cobertas com vegetação típica de Cerrado strictu sensu, conforme classificação fitofisionômica de Eiten (2001). Os critérios para escolha das áreas controles foram: i) a mesma classe de solo, e ii) a densidade arbórea da vegetação nativa situada próxima aos depósitos de resíduos domiciliares.

Nos depósitos de resíduos domiciliares estudados e nas áreas controles foram estabelecidas três parcelas de $25 \times 500 \mathrm{~m}$ de comprimento, que foram divididas em dez subparcelas de $25 \times 50 \mathrm{~m}$, onde foram coletados os dados de vegetação (Figura 1). O espaçamento entre as parcelas foi de $75 \mathrm{~m}$. Cada subparcela foi denominada com um número, e o mesmo procedimento foi realizado para a área controle onde a parte inicial das parcelas foi determinada de forma aleatória.

O levantamento da composição das espécies exóticas e nativas abrangeu todos os indivíduos arbóreos com diâmetro maior que $5 \mathrm{~cm}$, a $30 \mathrm{~cm}$ do solo (Chapman, 1977) localizados nas parcelas estabelecidas em áreas adjacentes de cada depósito de resíduos domiciliares e nas áreas determinadas para o controle (Figura 1).

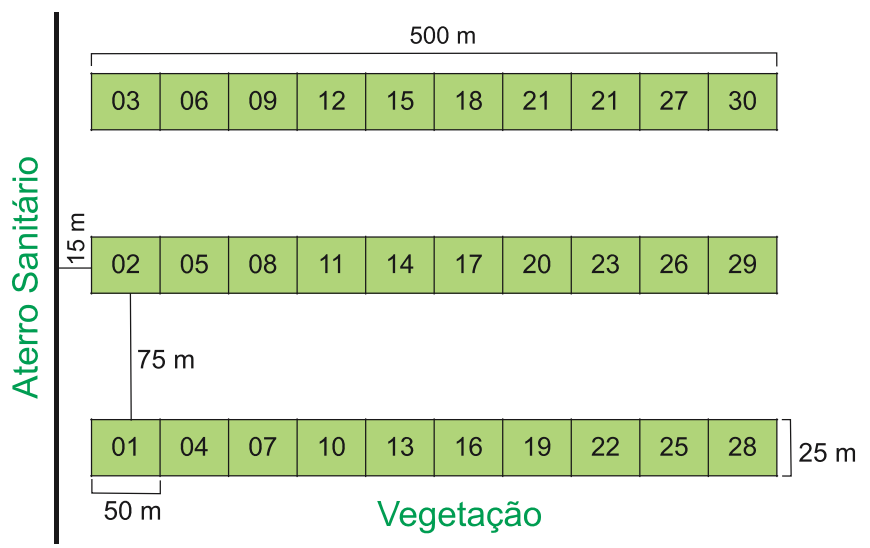

FIGURA 1: Esquema de distribuição das parcelas e subparcelas (1 a 30) de amostragem dos dados próximos aos depósitos de resíduos domiciliares e áreas controles (Adaptado de Carter, 1993 e Marguran, 1988).

As espécies arbóreas foram identificadas e seus diâmetros mensurados, com fita métrica (circunferência) e a altura medida com hipsômetro de Haga. A identificação foi feita pelo método de comparação de exsicatas (Breyer e Tsuboi, 1996) no Herbário UB e IBGE. Quando não se obteve a identificação, o material foi enviado a um especialista taxonômico. Os indivíduos arbóreos foram identificados por espécies, gênero e família pelo sistema APG II (Angiosperm Phylogeny Group II, 2003).

O Índice de Valor de Importância (IVI) de cada espécie nativa e exótica foi calculado segundo MuellerDumbois e Ellemberg (2002).

O impacto das plantas exóticas sobre as nativas foi calculado segundo o Índice de Impacto Ambiental de Exóticas (IIAE), efetuado a partir do cálculo dos coeficientes do impacto ambiental (Reaser et al., 2007). O cálculo foi realizado segundo a equação da página a seguir:

Este índice varia de -1 a 1 , sendo que -1 significa que a área não possui plantas nativas e 1 que a área não possui plantas exóticas. Segundo Reaser et al. (2007), valores abaixo de 0,8 já significariam uma preocupação para a biodiversidade local, pois 
representariam que cerca de $20 \%$ da área estaria ocupada pela vegetação exótica, necessitando uma intervenção e manejo local urgente.

$$
\text { IIAE }=-\frac{\left(\mathrm{P}_{\text {exóticas }}-\mathrm{P}_{\text {nativas }}\right)_{\text {subárea }}}{\frac{\mathrm{P}_{\text {total }}}{\mathrm{n}_{\text {área }}}}
$$

Sendo:

IIAE = índice de impacto ambiental de exóticas na subárea ou parcela estudada;

$\mathrm{P}_{\text {exóticas }}=$ valor do IVI das plantas exóticas na parcela ou no ponto de amostragem;

$\mathrm{P}_{\text {nativas }}=$ valor do IVI das plantas nativas na parcela ou no ponto de amostragem;

$\mathrm{P}_{\text {total }}=$ valor do IVI total (IVI $=300$ );

$\mathrm{n}_{\text {área }}=$ número de parcelas ou pontos de amostragem.

A forma de dispersão de sementes para as plantas encontradas foi classificada segundo Seghieri e Simier (2002) e Instituto Hórus (2007): anemocórica (dispersão pelo vento), autocórica (auto-dispersão, porém a planta não possui característica evidente de dispersão), barocórica (dispersão pela gravidade) e zoocórica (dispersão pelos animais). Especificamente, dentro da dispersão zoocórica tem-se a ornitocoria, dispersão por pássaros, e mirmecocoria, dispersão por formigas.

A classificação quanto ao tipo de planta exótica foi definida a partir da Convenção sobre Diversidade Biológica (CDB, 2008), conforme descrito na Tabela 1.

Ajustes da distribuição dos números de indivíduos pelas subparcelas, para determinação da reta e coeficiente de determinação $\left(\mathrm{R}^{2}\right)$, foram realizados pelo programa Statistica 5.0 (StatSoft, 1997), para observar possíveis padrões na distribuição dos indivíduos das espécies nativas e exóticas ao longo das subparcelas amostradas.
TABELA 1: Classificação quanto ao tipo de planta exótica.

\begin{tabular}{l|l|l}
\hline \multirow{2}{*}{$\begin{array}{l}\text { Espécies } \\
\text { exóticas }\end{array}$} & $\begin{array}{l}\text { São as espécies, subespécies ou taxa inferio- } \\
\text { res introduzidos fora da sua área natural de } \\
\text { distribuição presente ou passada, incluindo } \\
\text { qualquer parte, gametas, sementes, ovos ou } \\
\text { propágulos dessas espécies que possam so- } \\
\text { breviver e posteriormente reproduzir-se. }\end{array}$ \\
\hline i) Contidas & $\begin{array}{l}\text { São as espécies cujo poten- } \\
\text { cial de invasão é reconheci- } \\
\text { do pelo histórico de invasão } \\
\text { em outros locais, porém que } \\
\text { se encontra com a dispersão } \\
\text { limitada em função do uso a } \\
\text { que se destina, por controle } \\
\text { humano. }\end{array}$ \\
\hline \multirow{2}{*}{$\begin{array}{l}\text { Classificação } \\
\text { de espécies } \\
\text { exóticas }\end{array}$} & $\begin{array}{l}\text { São as espécies que não } \\
\text { têm inimigos naturais para } \\
\text { limitar sua reprodução e } \\
\text { disseminação, resultando } \\
\text { elevado potencial de colo- } \\
\text { nização, ameaçando ecos- } \\
\text { sistemas, hábitats e espécies } \\
\text { nativas. }\end{array}$ \\
\hline & $\begin{array}{l}\text { São as espécies cujo poten- } \\
\text { cial de invasão é reconheci- } \\
\text { do pelo histórico de invasão } \\
\text { em outros locais, porém } \\
\text { sem expressão de invasão, } \\
\text { ou seja, em estado latente. }\end{array}$ \\
\hline $\begin{array}{l}\text { São as espécies cujo poten- } \\
\text { cial de invasão é reconheci- } \\
\text { do pelo histórico de invasão } \\
\text { em outros locais, já em está- } \\
\text { gio de auto-regeneração em } \\
\text { nível local. }\end{array}$ \\
\hline
\end{tabular}

Fonte: CDB (2008).

\section{Resultados e Discussão}

Vinte e três espécies exóticas foram encontradas nas subparcelas de estudo, distribuídas em 13 famílias (Tabelas 2 e 3). Apesar do grande número de espécies exóticas encontradas, poucos indivíduos foram amostrados para cada espécie. 
TABELA 2: Número de espécies e famílias nativas de Cerrado e exóticas nas áreas de estudos adjacentes ao aterro do Jockey Club de Brasília (BSB) e do Aterro Sanitário de Goiânia (GYN), e em duas áreas controles estabelecidas (BSB - Controle e GYN Controle).

\begin{tabular}{c|c|c|c}
\hline \multirow{2}{*}{ BSB } & & Espécies & Famílias \\
\hline \multirow{2}{*}{ BSB - Controle } & Nativas & 70 & 30 \\
\cline { 2 - 4 } & Exóticas & 10 & 8 \\
\cline { 2 - 4 } & Nativas & 71 & 39 \\
\cline { 2 - 4 } GYN & Exóticas & 3 & 2 \\
\cline { 2 - 4 } & Nativas & 73 & 36 \\
\hline \multirow{2}{*}{ GYN - Conóticas } & 12 & 11 \\
\hline
\end{tabular}

Das 252 espécies exóticas levantadas por Pereira e Filgueiras (1988) em áreas de Cerrado, 47 eram espécies arbóreas-arbustivas. Oficialmente no Brasil, foram catalogadas 55 espécies arbóreo-arbustivas exóticas (Instituto Hórus, 2007). Neste trabalho foram registrados aproximadamente $50 \%$ do número de espécies registradas.

A espécie que obteve maior número de indivíduos foi Ricinus communis (Tabela 3), com oito indivíduos nas parcelas estudadas próximo ao aterro Sanitário de Goiânia. Nas subparcelas estudadas próximo ao aterro do Jockey Club de Brasília, Citrus limon, com quatro indivíduos, e Tecoma stans, com três, foram as espécies com maiores números de indivíduos.

TABELA 3: Composição das espécies exóticas, suas respectivas famílias e número de indivíduos encontrados nas áreas de estudos adjacentes ao aterro do Jockey Club de Brasília (BSB) e do Aterro Sanitário de Goiânia (GYN), e em duas áreas controles estabelecidas (BSB - Controle e GYN - Controle).

\begin{tabular}{|c|c|c|c|c|c|}
\hline \multirow{3}{*}{ Espécie Exótica } & \multirow{3}{*}{ Família } & \multicolumn{4}{|c|}{ Número de indivíduos } \\
\hline & & \multicolumn{2}{|c|}{ Aterros } & \multicolumn{2}{|c|}{ Controles } \\
\hline & & BSB & GYN & BSB & GYN \\
\hline Acacia mearnsii De Wild. & Fabaceae & 2 & - & - & - \\
\hline Casuarina equisetifolia $\mathrm{L}$. & Casuarinaceae & - & 3 & - & - \\
\hline Citrus aurantium $\mathrm{L}$. & Rutaceae & - & 1 & - & - \\
\hline Citrus limon (L.) Burm. f. & Rutaceae & 4 & - & - & - \\
\hline Eucalyptus grandis W. Hill & Myrtaceae & 1 & - & - & - \\
\hline Eucalyptus robusta $\mathrm{Sm}$. & Myrtaceae & - & 1 & - & - \\
\hline Leucaena leucocephala (Lam.) de Wit & Fabaceae & 1 & - & - & - \\
\hline Ligustrum japonicum Thunb. & Oleaceae & - & 2 & - & - \\
\hline Ligustrum lucidum W. T. Aiton & Oleaceae & 1 & - & - & - \\
\hline Melia azedarach $\mathrm{L}$. & Meliaceae & - & 1 & - & - \\
\hline Morus nigra L. & Moraceae & - & 1 & - & - \\
\hline Musa ornata Roxb. & Musaceae & - & 1 & - & - \\
\hline Musa rosacea Jacq. & Musaceae & - & 2 & - & - \\
\hline Pinus caribaea Morelet & Pinaceae & - & - & 1 & - \\
\hline Pinus elliottii Engelm. & Pinaceae & - & 1 & - & - \\
\hline Pinus taeda var. heterophylla Elliott & Pinaceae & 1 & - & - & - \\
\hline Pittosporum undulatum Vent. & Pittosporaceae & - & 1 & - & - \\
\hline Psidium guajava $\mathrm{L}$. & Myrtaceae & 1 & - & 1 & - \\
\hline Ricinus communis L. & Euphorbiaceae & - & 8 & - & - \\
\hline Spathodea campanulata P. Beauv. & Bignoniaceae & - & 1 & - & - \\
\hline Syzygium cumini (L.) Skeels & Myrtaceae & 1 & - & - & - \\
\hline Tecoma stans (L.) Juss. ex Kunth & Bignoniaceae & 3 & - & 1 & - \\
\hline Terminalia catappa $\mathrm{L}$. & Combretaceae & 2 & - & - & - \\
\hline
\end{tabular}


Ricinus communis, popularmente chamada de "mamona", utilizada principalmente para produção do biodiesel e extração do óleo, desenvolve-se em praticamente todos os tipos de solos brasileiros, com preferência de solos férteis e bem drenados, em regiões com precipitação anual próximas de $700 \mathrm{~mm}$ (Távora, 1982). Essa espécie tem uma maior incidência em áreas perturbadas de Cerrado, principalmente pelo despejo de resíduos provenientes da construção civil (Távora, 1982). Sua dispersão foi descrita sendo autocórica (Seghieri e Simier, 2002; Brighenti et al., 2001), e também mirmecocórica. (Brighenti et al., 2001).

Citrus limon, popularmente conhecido por limoeiro, é freqüentemente utilizado na dieta alimentar humana $\mathrm{e}$ por isso bastante propagada pelo Brasil e pelo mundo. No Cerrado a sua disseminação está principalmente atribuída ao homem (Instituto Hórus, 2007). A presença dessa espécie em áreas adjacentes a depósito de resíduos domiciliares pode se atribuir aos resíduos domiciliares, que contêm as sobras com suas sementes.

Tecoma stans, conhecido como ipê-de-jardim, é utilizado para projetos de arborização, urbanização e paisagísticos (Instituto Hórus, 2007). Apesar de ser bastante vistosa, esta espécie exótica abafa a vegetação nativa por formar aglomerados densos, retardando a regeneração natural em áreas degradadas, diminuindo a biodiversidade e inutilizando pastos (Lorenzi, 2000).

Estas e as espécies amostradas têm síndromes de dispersão de sementes favoráveis (Tabela 4), pois a maioria delas são anemocóricas, ou seja, têm sua dispersão de sementes pelo vento, o que permitem uma propagação por grandes distâncias da árvore de origem (árvore-mãe). Animais (zoocoria), particularmente pássaros (ornitocoria), favorecem também esta propagação a longas distâncias.

Além destes tipos de dispersão, outras espécies relatadas como Citrus aurantium possui síndrome de dispersão por barocoria, que é por gravidade, o que dificulta a sua distribuição por grandes distâncias de suas origens, a menos que também haja distribuição zoocórica. Colautti et al. (2005) observaram que estas espécies foram introduzidas em parques e em unidades de conservação pelo próprio homem, diretamente pelo cultivo de plantas para ornamentos ou indiretamente, pelo consumo de frutas nesses locais ou o transporte de sementes, sem perceber, aderidas em carros ou nas roupas.

Ainda que nem todas as espécies observadas apresentem comportamento invasor nos locais estudados, todas elas têm potencial de invasão em função de seu histórico em outros locais. Todas constam na base de dados nacional de espécies exóticas invasoras (Instituto Hórus, 2007).

Nas subparcelas estudadas, tomaram-se em consideração vários fatores que permitiram explicar o aparecimento das espécies amostradas: $1^{\circ}$ ) pela forma de dispersão; $2^{\circ}$ ) pela introdução de espécies exóticas, como Pinus e Eucalyptus, para a formação de uma barreira de vegetação, evitando-se a propagação do odor, causado pelos resíduos sólidos e pelo chorume; 3) pelo trânsito de pessoas e carros próximos aos depósitos de resíduos domiciliares, podendo trazer sementes a estes locais; 4ㅇ) pelos próprios resíduos sólidos e lixos domiciliares conterem resto de frutas, solos e xaxim, que podem trazer sementes e ajudar a disseminar plantas exóticas; e 5ㅇ) a restos de limpeza e podas em jardins e parques, que são destinados a estes depósitos. Blumenthal (2005) corrobora com estes fatores e culpa principalmente o homem, direta ou indiretamente, pela invasão de plantas exóticas em habitats naturais.

A Figura 2 mostra a diferença de padrão de distribuição das espécies nativas e exóticas nas subparcelas estudadas. As espécies nativas se distribuem de forma eqüitativa, representada pela reta do ajuste (Figura 2A), enquanto as espécies exóticas possuem um número maior de indivíduos nas subparcelas mais próximas aos depósitos de resíduos domiciliares do que em subparcelas mais distantes (Figura 2B), padrão não observado nas áreas controles. 
TABELA 4: Forma de dispersão natural de sementes e classificação quanto ao comportamento de invasão das espécies amostradas nas áreas de estudos adjacentes ao aterro do Jockey Club de Brasília (BSB) e do Aterro Sanitário de Goiânia (GYN), e em duas áreas controles estabelecidas (BSB - Controle e GYN - Controle).

\begin{tabular}{l|c|c}
\multicolumn{1}{c|}{ Espécie } & Forma de Dispersão* & Classificação** \\
\hline Acacia mearnsii & Anemocórica/Ornitocórica & Est. \\
\hline Casuarina equisetifolia & Anemocórica & Inv. \\
\hline Citrus aurantium & Barocórica & Est./Cont. \\
\hline Citrus limon & Barocórica & Est. \\
\hline Eucalyptus grandis & Anemocórica & Intr./Cont. \\
\hline Eucalyptus robusta & Anemocórica & Intr. \\
\hline Leucaena leucocephala & Zoocórica & Inv. \\
\hline Ligustrum japonicum & Ornitocórica \\
\hline Ligustrum lucidum & Ornitocórica & Inv. \\
\hline Melia azedarach & Ornitocórica & Inv. \\
\hline Morus nigra. & Ornitocórica & Inv. \\
\hline Musa ornata & Autocórica/Zoocórica & Inv. \\
\hline Musa rosacea & Autocórica/Zoocórica & Inv. \\
\hline Pinus caribaea & Anemocórica & Intr. \\
\hline Pinus elliottii & Anemocórica & Intr. \\
\hline Pinus taeda var. heterophylla & Anemocórica & Intr. \\
\hline Pittosporum undulatum & Ornitocórica/Barocórica & Inv. \\
\hline Psidium guajava & Ornitocórica & Inv. \\
\hline Ricinus communis & Autocórica/Mirmecocórica & Inv. \\
\hline Spathodea campanulata & Anemocórica & Inv. \\
\hline Syzygium cumini & Ornitocórica & Inv. \\
\hline Tecoma stans & Anemocórica & Inv. \\
\hline Terminalia cattapa & Autocórica/Zoocórica & Inv. \\
\hline Fon Segric & \\
\hline
\end{tabular}

* Fonte: Seghieri e Simier (2002); Instituto Hórus (2007); **Classificação e Fonte: CDB (2008).

(Est. = estabelecidas; Inv. = invasoras; Int. = introduzidas; Cont. = contidas).

A Figura 2 corroborou com as observações de D'Antonio e Kark (2002), que registraram uma maior quantidade de indivíduos de espécies exóticas, em áreas de vegetação nativa próximas aos centros urbanos, do que em áreas distantes.

Observa-se, assim, a influência da presença dos depósitos de resíduos domiciliares na distribuição das plantas exóticas, confirmada também, pela porcentagem do índice de valor de importância (IVI) das plantas exóticas em relação ao IVI total (IVI das plantas nativas + IVI das plantas exóticas). A contribuição das plantas exóticas no IVI se mostra maior nas subparcelas mais próximas dos depósitos de resíduos domiciliares (Figura 3).

Corroborando com a figura 3, quando se calculou o índice de impacto ambiental de exóticas (IIAE - Figura 4), os valores de IIAE mostraram que o maior impacto das espécies exóticas sobre espécies nativas ocorreram em áreas mais próximas dos depósitos de resíduos domiciliares, apresentando valores entre 0,995 a 0,998 , do que em áreas mais distantes.

Reaser et al. (2007) obtiveram valores negativos $(-0,5)$ em algumas áreas, onde mais de $75 \%$ da área de estudo (em ilhas) fora dominado por plantas exóticas. Genovesi (2005) também descreveu altos valores de índices de impacto ambiental por espécies exóticas em várias áreas da Europa. Os dois autores salientaram que o índice representa a situação atual da invasão biológica, não podendo prever futuro potencial invasor na comunidade vegetal, sendo que a presença de indivíduos das espécies exóticas em qualquer ambiente já representa uma preocupação de manejo ambiental. 


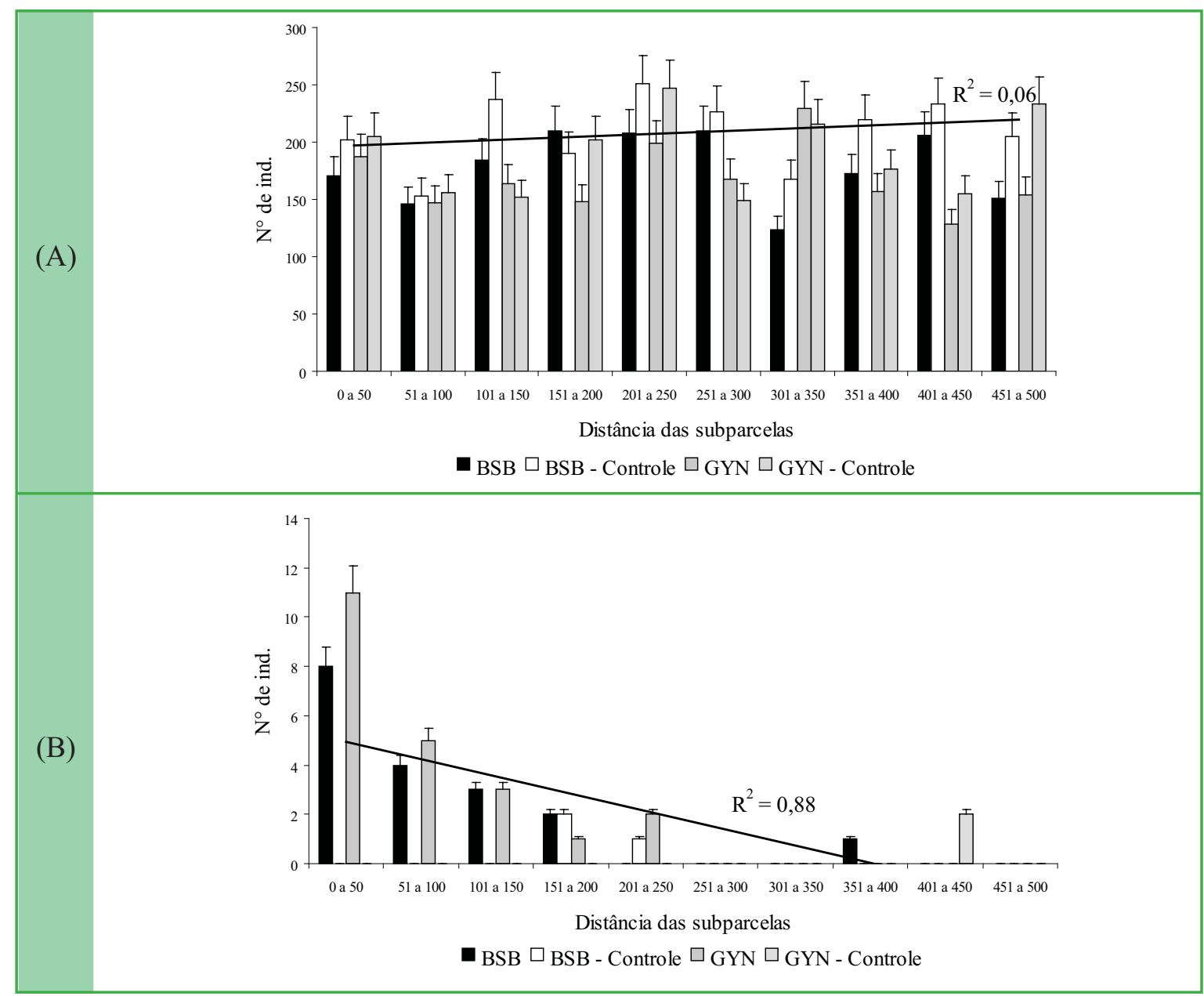

FIGURA 2: Distribuição dos indivíduos das espécies nativas (A) e das espécies exóticas (B) nas áreas de estudos adjacentes ao aterro do Jockey Club de Brasília (BSB) e do Aterro Sanitário de Goiânia (GYN), e em duas áreas controles estabelecidas (BSB Controle e GYN - Controle). A linha corresponde ao ajuste linear do número de indivíduos pela distância das subparcelas e $\mathrm{R}^{2}=$ coeficiente de determinação do ajuste.

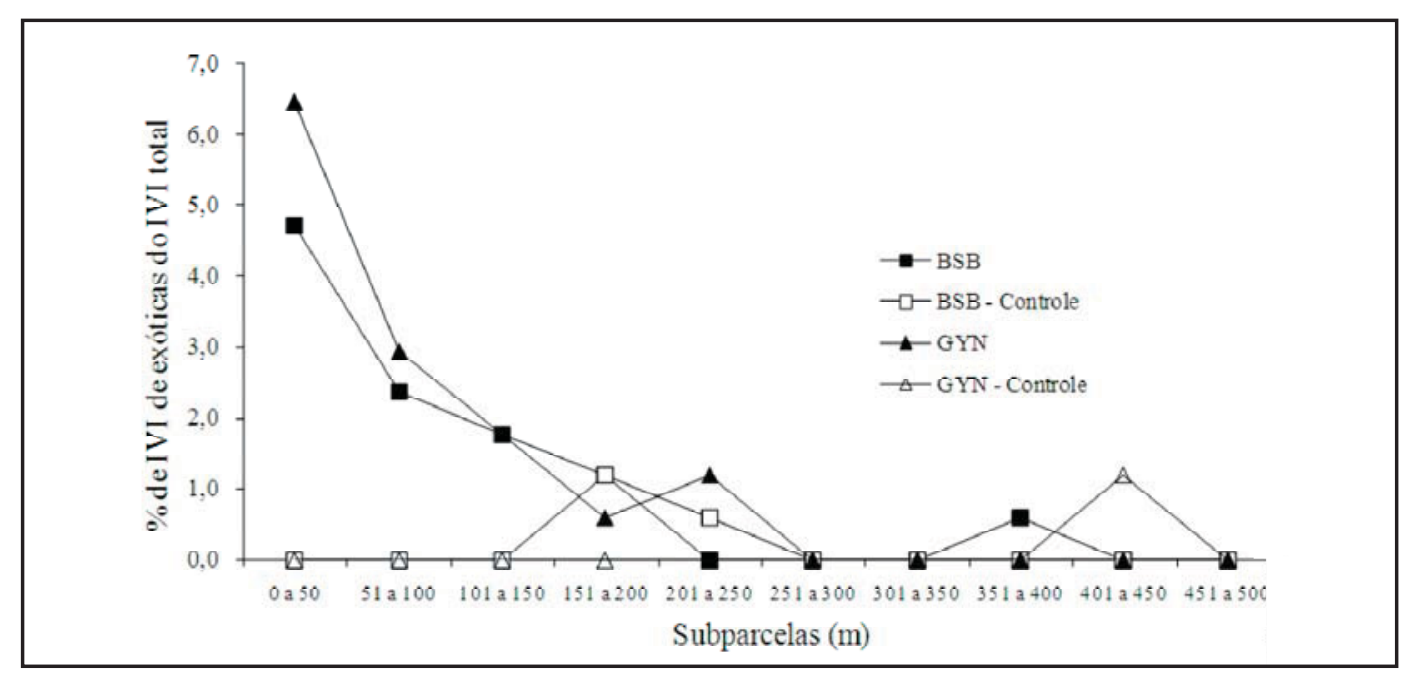

FIGURA 3: Porcentagem do índice de valor de importância (IVI) de plantas exóticas do IVI total nas áreas de estudos adjacentes ao aterro do Jockey Club de Brasília (BSB) e do Aterro Sanitário de Goiânia (GYN), e em duas áreas controles estabelecidas (BSB - Controle e GYN - Controle). 


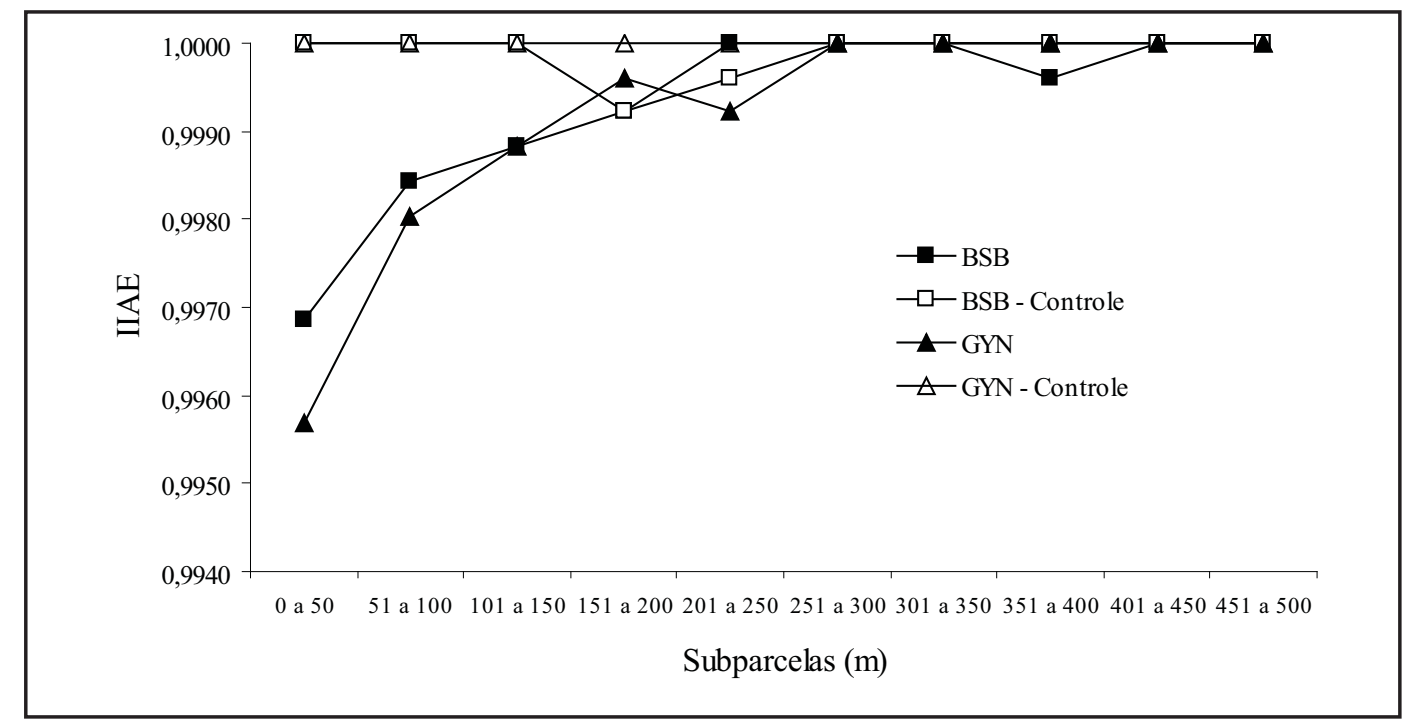

FIGURA 4: Índice de impacto ambiental de plantas exóticas (IIAE) pelas distâncias das parcelas estudadas nas áreas de estudos adjacentes ao aterro do Jockey Club de Brasília (BSB) e do Aterro Sanitário de Goiânia (GYN), e em duas áreas controles estabelecidas (BSB - Controle e GYN - Controle).

Kolar e Lodge (2002) e Leung et al. (2002) também discutiram em seus trabalhos a importância dos índices de avaliação ambiental para o alerta de manejo e monitoramento das plantas exóticas sobre as nativas, porém deixaram evidente que a presença de um único indivíduo de planta exótica poderá ser suficiente em longo e médio prazos para a disseminação da espécie em ambientes naturais.

Nas áreas amostradas situadas próximo aos depósitos de resíduos domiciliares, poucos indivíduos de plantas exóticas foram amostrados, porém os indivíduos arbóreos estavam bem estabelecidos, como é o caso de uma árvore de Pinus elliottii, em Goiânia, com $24 \mathrm{~m}$ de altura e $30 \mathrm{~cm}$ de diâmetro.

Lorenzi (2000), Lima (2003), Blumenthal (2005) e Filgueiras (2005) alertam que países tropicais são excelentes hábitats para as plantas exóticas, principalmente o Brasil, pois além de fornecer clima e substrato suscetíveis à sua propagação, as espécies exóticas estão livres de competidores, predadores e parasitas, apresentando vantagens fitofisiológicas competitivas com relação a espécies nativas.

No Cerrado esta preocupação é ainda maior, pois, as práticas erradas de manuseio dos ecossistemas, como a remoção de áreas florestais, queimadas anuais para preparo da terra, erosão e pressão excessiva de pastoreio contribuem para a perda de diversidade natural e fragilidade do meio a invasões (Ziller, 2000; Hoffmann et al., 2004).

Pode-se concluir, então, com este trabalho: i) 22 espécies exóticas foram encontradas nas parcelas estudadas próximos aos depósitos de resíduos domiciliares distribuídas em 13 famílias, e apenas 2 espécies foram encontradas nas áreas controle; e ii) a presença de depósitos de resíduos domiciliares aumenta a incidência de espécies exóticas arbóreas, causando um gradiente na distribuição de seus indivíduos, padrão não observado nas áreas controles.

\section{Referências}

Angiosperm Phylogeny Group II - APG II. 2003. An update of the angiosperm phylogeny group classification for orders and families of flowering plants: APG II. Botanic Journal Linnean Society, 141: 399-436.

Blumenthal, D. 2005. Interrelated causes of plant invasion. Science, 310: 243-244.

Breyer, L. M.; Tsuboi, E. 1996. Sistema herbário: Manual de operações. Editora Universidade de Brasília, Brasília, Brasil, 109pp.

Brighenti, A. M. Biologia de plantas daninhas. In: Oliveira Júnior, R. S.; Constantin, J. (Eds). Plantas daninhas e seu manejo. Guaíba: Agropecuária, 2001. p. 18-58.

Carter, M. R. 1993. Soil sampling and methods of analysis. Lewis, Boca Raton, USA, 823pp. 
CDB - Convention on Biological Diversity. 2008. CDB Handbook. $3^{\text {rd }}$ ed. Disponível em $<$ http://www.cbd.int/handbook/>. Acesso em 14 de abril de 2008.

Chapman, S. B. 1977. Methods in plant ecology. The Journal of Ecology, 65 (2): 718-719.

Colautti, R. I.; Muirhead, J. R.; Biswas, R. N.; MacIssac, H. J. 2005. Realized versus apparent reduction in enemies of the European starling. Biological Invasions, 7: 723-732.

D'Antonio, C. M.; Kark, S. 2002. Impacts and extent of biotic invasions in terrestrial ecosystems. Trends in Ecology and Evolution, 17: 202-204.

Eiten, G. 2001. Vegetação natural do Distrito Federal. SEBRAE, Brasília, Brasil, 162pp.

Embrapa - Empresa Brasileira de Pesquisa Agropecuária e Centro Nacional de Pesquisa de Solos (Rio de Janeiro, RJ). 1999. Sistema Brasileiro de Classificação de Solos. Embrapa Produção da Informação e Embrapa Solos, Brasília e Rio de Janeiro, Brasil, 412pp.

Filgueiras, T. S. 2005. Asiáticas no Brasil: Gramíneas (Poaceae) introduzidas da Ásia. Eugeniana, 28: 3-18.

Filgueiras, T. S. 1990. Africanas no Brasil: Gramíneas introduzidas na África. Cadernos de Geociências, 5: 57-63.

Instituto Hórus. 2007. Espécies exóticas invasoras: Fichas técnicas. Disponível em <http://www.institutohorus.org.br/inf_fichas.htm>. Acesso em 21 de janeiro de 2007.

IUCN - International Union for conservation of nature and natural resources. 2000. IUCN guidelines for the prevention of biodiversity loss caused by alien invasive species. $\mathbf{5 1}^{\text {st }}$ Meeting of Council, Gland, Switzerland, p.18.

Genovesi, P. 2005. Eradications of invasive alien species in Europe: a review. Biological Invasions, 7: 127-133.

Gotelli, N. J.; Colwell, R. K. 2001. Quantifying biodiversity: procedures and pitfalls in the measurement and comparison of species richness. Ecology Letters, 4: 379-391.

Hoffmann, W. A.; Lucatelli, V. M. P. C.; Silva, F. J.; Azeuedo, I. N. C.; Marinho, M. da S.; Albuquerque, A. M. S.; Lopes, A. de O. e Moreira, S. P. 2004. Impact of the invasive grass Melinis minutiflora at the savanna-forest ecotone in the Brazilian Cerrado. Diversity and Distributions, 10: 99-103.

Kolar, C. S.; Lodge, D. M. 2002. Ecological predictions and risk assessment for alien fishes in North America. Science, 298: 12331236.

Leung, B.; Lodge, D. M.; Finnoff, D.; Shogren, J. F.; Lewis, M. A.; Lamberti, G. 2002. An ounce of prevention or a pound of cure: bioeconomic risk analysis of invasive species. Proceedings of the Royal Society of London, 269: 2407-2413.

Lima, L. 2003. Espécies invasoras. Revista Galileu, 145: 45-56.

Lorenzi, H. 2000. Plantas daninhas do Brasil: Terrestres, aquáticas, parasitas e tóxicas. $3^{\mathrm{a}}$ ed. Nova Odessa, São Paulo, Brasil, 69pp.

Mack, R. N.; Chair; S. D.; Lonsdale, W. M.; Evans, H.; Clout, M.; Bazzaz, F. 2000. Biotic invasions: causes, epidemiology, global consequences and control. Issues in Ecology, 5: 1-20.
Marguran, A. E. 1988. Ecological diversity and its measurement. $2^{\text {nd }}$ ed. Princeton University Press, Princeton, USA, 179pp.

Mueller-Dombois, D.; Ellemberg, H. 2002. Aims and methods of vegetation ecology. John Wiley and Sons, New York, USA, $547 \mathrm{pp}$.

Parker, I. M.; Simberloff, D.; Lonsdale, W. M.; Goodell, K.; Wonham, M.; Kareiva, P. M.; Williamson, M. H.; Holle, B. V.; Moyle, P. B.; Byers, J. E.; Goldwasser, L. 1999. Impact: toward a framework for understanding the ecological effects of invaders. Biological Invasions, 1: 3-19.

Pereira, B. A. S.; Filgueiras, T. S. 1988. Levantamento qualitativo das espécies invasoras da Reserva Ecológica do IBGE, Brasília (DF), Brasil. Cadernos de Geociências, 1: 29-38.

Prefeitura de Goiânia. 2007. Disponível em: <www.goiania.go.gov. br>. Acesso em 03 de julho de 2007.

Randall, J. M. 1996. Weed control for the preservation of biological diversity. Weed technology, 10: 370-383.

Reaser, J. K.; Meyerson, L. A.; Cronk, Q.; Poorter, M.; Eldrege, L. G.; Green, E.; Kairo, M.; Latasi, P.; Mack, R. N.; Mauremootoo, J.; O'dowd, D.; Orapa, W.; Sastroutomo, S.; Saunders, A.; Shine, C.; Thrainsson, S.; Vaiutu, L. 2007. Ecological and socioeconomic impacts of invasive alien species in island ecosystems. Environmental Conservation, 34 (2): 98-111.

Santana, O. A.; Imaña-Encinas, J. 2004. Modelo espacial de contaminação do solo e do lençol freático do aterro do Jockey Club para o Parque Nacional de Brasília. Brasília - DF. In: Pejon, O. J. \& Zuquette, L. V. (Eds). Cartografia Geotécnica e Geoambiental (Conhecimento do Meio Físico: Base para a Sustentabilidade). Ed. ABGE/UFSCAR, São Carlos, Brasil, p.453-460.

Santos, H. G.; Coelho, M. R.; Anjos, L. H. C.; Jacomine, P. K. T.; Oliveira, V. Á.; Lumbreras, J. F.; Oliveira, J. B.; Carvalho, A. P.; Fasolo, P. J. 2003. Propostas de revisão e atualização do Sistema Brasileiro de Classificação de Solos. Embrapa Solos (Embrapa Solos. Documentos; n. 53), Rio de Janeiro, Brasil, 56pp.

Seduh - Serviço de Limpeza Urbana (DF). 2007. Disponível em <www.slu.df.gov.br>. Acesso em 03 de janeiro de 2007.

Seghieri, J.; Simier, M. 2002. Variations in phenology of a residual invasive shrub species in Sahelian fallow savannas, south-west $\mathrm{Ni}$ ger. Journal of Tropical Ecology, 18: 897-912.

StatSoft. 1997. Statistic for Windows 5.1. StatSoft Inc., Tulsa, USA, CD Rom.

Távora, F. J. F. 1982. A cultura da mamona. EPACE, Fortaleza, Brasil, 59pp.

Westbrooks, R. 1998. Invasive plants: changing the landscape of America: fact book. Federal Interagency Committee for the Management of Noxious and Exotic Weeds, Washington, USA, 107pp.

Zanchetta, D.; Diniz, F. V. 2006. Estudo da contaminação biológica por Pinus spp. em três diferentes áreas na estação ecológica de Itirapina (SP, Brasil) Revista do Instituto Florestal, 18: 1-14.

Ziller, S. R. 2000. A Estepe Gramíneo-Lenhosa no segundo planalto do Paraná: diagnóstico ambiental com enfoque à contaminação biológica. Tese de Doutorado, Universidade Federal do Paraná, Brasil, 268pp. 